\title{
OBSERVATIONAL PROPERTIES OF HELICAL MAGNETIC FIELDS
}

\author{
M. URBANIK and K. OTMIANOWSKA-MAZUR \\ Astronomical Observatory, Jagiellonian University \\ ul. Orla 171, Krakow, Poland
}

November 17, 1992

\begin{abstract}
The model of the polarized emission from a spiral galaxy similar to M31, containing helically twisted magnetic field has been constructed. Helical magnetic field was found to cause a specific asymmetry of the distribution of the polarized brightness of the galactic disk.
\end{abstract}

Key words: -galaxies - magnetic fields - polarized emission

\section{Introduction}

Helical magnetic fields are often implied by solutions of dynamo equations (e.g. Donner and Brandenburg, 1990) and by simulations of magnetic field structures in a turbulent medium (Otmianowska-Mazur and Urbanik, this volume). In order to explore possible observational consequences of the existence of such field configurations in galactic disks we constructed the model of the polarized emission from a M31-like galaxy, with the radio emission concentrated in a single ring. A comparison of the model with observations has been made, too.

\section{Model description}

The emission ring of the model galaxy was assumed to be composed of two helical magnetic tubes: one running above and one below the disk plane, with an opposite sense of the field twisting (see Otmianowska-Mazur et al., 1992). The density of cosmic ray electrons was assumed to decrease with the distance from the ring center and from the galactic plane as a two-dimensional Gaussian function. The pitch angle of the field twisting $\psi$, the tube cross-section radius and the cosmic-ray electron radial and vertical scales were the variable model parameters.

\section{Results}

Fig. 1 shows a typical map of the polarized emission from our model galaxy. It has been obtained for the field pitch angle corresponding to $\tan (\psi)=0.7$, the tube radius of $100 \mathrm{pc}$ and for the cosmic ray scales of $1.5 \mathrm{kpc}$ and $350 \mathrm{pc}$ in radial and vertical directions, respectively. The galaxy inclination angle was $77^{\circ}$, similar to that of M31. A specific asymmetry visible in the Figure, namely horizontal shifts in opposite directions of polarized emission maxima, was found to occur over a wide range of model parameters. The effect disappears when a mixture of an azimuthal and purely random field is assumed, thus it is a typical property of helical magnetic fields.

The degree of the asymmetry (measured by subtracting the flux integrated in the first and third disk quadrant from that in the second and fourth ones) 


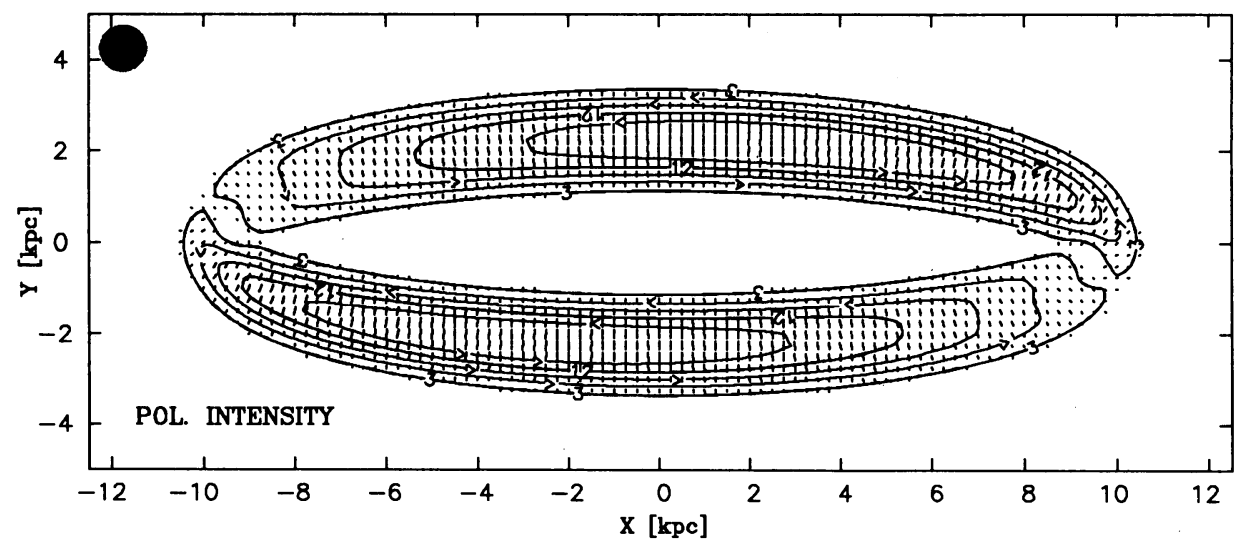

Fig. 1. The contours and E-vectors of the polarized intensity for the model galaxy (see text for model parameters). The model was made in arbitrary units in which the peak total power flux is 100 . The assumed telescope beam is marked in the upper left corner.

was found to increase with the field pitch angle and with the tube size, and to decrease with increasing vertical electron scale. The asymmetry depends also on the galaxy inclination angle: it is absent in perfectly edge-on and face-on galaxies being strongest for the inclination angle of about $80^{\circ}$.

The observations of the real M31 galaxy at the wavelength of $11 \mathrm{~cm}$ (Beck, 1982 ) revealed the asymmetry of the polarized emission similar to that obtained from our model. For a more detailed comparison with observations we constructed the model of this object containing 36 helical flux tubes with some admixture of a purely random field. The ratio of both magnetic field components, as well as the cosmic-ray electron density were assumed to vary azimuthally with the star formation rate, the latter being measured by the far-infrared emission (Walterbos and Schwering, 1987). A reference model with a mixture of azimuthal and random fields was considered as well. The assumption of the helical magnetic field was found to yield a much better agreement between the model and observations than in the case of no field twisting. A more detailed discussion will be published in a separate paper (Urbanik et al., 1992).

This work was supported by the grant from the Polish Committee for Scientific Research (KBN), project no. 2.1172.91.01

\section{References}

Beck, R.: 1982, Astron. Astrophys. 106, 121

Donner, K.J., Brandenburg, A.: 1990, Astron. Astrophys. 240, 289

Otmianowska-Mazur, K., Urbanik, M., Terech, A.: 1992, Geophys. Astrophys. Fluid Dyn , (in press)

Urbanik, M., Otmianowska-Mazur, K., Beck, R.: 1992, Astron. Astrophys , (submitted)

Walterbos, R.A.M., Schwering, P.B.W.: 1987, Astron. Astrophys. 180, 27 